\title{
Noninvasive surveillance for cardiac allograft vasculopathy following heart transplantation: One of several emerging clinical applications for cardiac positron emission tomography with assessment of myocardial blood flow reserve
}

\author{
Dennis A. Calnon, MD, MASNC \\ a OhioHealth Heart and Vascular Physicians, Columbus, $\mathrm{OH}$
}

Received Aug 2, 2021; accepted Aug 3, 2021

doi: 10.1007/s12350-021-02776-7

\section{See related article, pp. 2555-2567}

Cardiac positron emission tomography (PET) with assessment of myocardial blood flow reserve (MBFR) has matured over several decades and is now widely recognized as a powerful tool for the diagnosis of obstructive coronary artery disease (CAD), for the assessment of prognosis in patients with known or suspected $\mathrm{CAD}$, and for informing decisions for revascularization. ${ }^{1}$ In addition to the assessment of obstructive epicardial coronary artery atherosclerosis, cardiac PET with assessment of MBFR is capable of comprehensively evaluating all levels of the vascular supply to the myocardium (large conduit epicardial arteries, resistance arterioles, and the microvasculature) and has demonstrated the potential to provide useful information in other clinical scenarios.

(1) Identification of coronary microvascular disease as an etiology for anginal symptoms in the absence of obstructive CAD. ${ }^{2}$

(2) Assessment of patients with hypertrophic cardiomyopathy $^{3}$ and other nonischemic cardiomyopathies. ${ }^{4}$

Reprint requests: Dennis A. Calnon, MD, MASNC, OhioHealth Heart and Vascular Physicians, 3705 Olentangy River Road, Suite 100, Columbus, OH 43214; Dennis.Calnon@OhioHealth.com

J Nucl Cardiol 2022;29:2568-70.

$1071-3581 / \$ 34.00$

Copyright (c) 2021 American Society of Nuclear Cardiology.
(3) Assessment of patients with asymptomatic aortic stenosis with potential to inform decisions regarding timing of valve interventions. ${ }^{5}$

(4) Assessment of patients with cardiac amyloidosis with potential to determine likelihood of response to medical therapy. ${ }^{6}$

(5) Noninvasive diagnostic and prognostic assessment of cardiac allograft vasculopathy (CAV) following heart transplantation (HTx). ${ }^{7-12}$

Cardiac PET with assessment of MBFR seems to be ideally suited for the evaluation of CAV. CAV is a diffuse process affecting the epicardial coronary arteries and the microvasculature resulting in reduced vasodilatory capacity. Cardiac PET with assessment of MBFR is noninvasive, safe in patients with renal failure, feasible in patients with arrhythmias or elevated resting heart rates, feasible in patients with implanted pacemakers or defibrillators, and capable of detecting diffuse reductions in coronary vasodilatory capacity due to CAV.

The study by Nelson and colleagues in this issue ${ }^{13}$ adds to the existing literature supporting the clinical utility of cardiac PET with assessment of MBFR for the noninvasive assessment of CAV following HTx. The diagnosis of CAV can be challenging due to the diffuse (rather than focal) luminal narrowing characteristic of this condition and the lack of typical anginal symptoms in patients with denervated hearts following cardiac transplantation. The diagnosis of CAV is critically important due to the availability of treatments and the poor prognosis of CAV if unrecognized and untreated. Invasive coronary angiography (ICA) with intravascular ultrasound (IVUS) has traditionally been used for routine surveillance, though this procedure is invasive and 
is associated with a small but finite risk for complications including contrast-induced nephropathy (CIN) and vascular injury. Similarly, coronary computed tomography angiography (CCTA) is contraindicated in the setting of renal dysfunction and is unable to assess the functional significance of diffuse coronary arterial narrowing. Conventional stress imaging modalities such as single-photon emission computed tomography (SPECT) myocardial perfusion imaging and stress echocardiography are not ideally suited for assessment of CAV due to the diffuse (rather than regional) abnormalities of myocardial blood flow in this condition.

The study by Nelson and colleagues was a retrospective single-center study involving 50 patients who had PET MBFR measurements performed for routine surveillance at a median of 10 (6.7 to 16) years after HTx. Patients were grouped according to MBFR $\leq 2$ (n $=25)$ or MBFR $>2(n=25)$. The authors found that during a median follow-up of 3.6 years, the probability of event-free survival was significantly higher in those with normal MBFR compared to those with low MBFR. The patients in the study by Nelson and colleagues were referred for cardiac PET primarily due to contraindications to ICA and IVUS (renal failure, arterial access limitations). Therefore, renal failure was common (estimated GFR $<30$ in $20 \%$ of patients). The primary outcome was all-cause mortality though secondary outcomes were assessed as well (revascularization, graft failure, retransplantation, and new CAV diagnosis).

A strength of the study was the relatively long-term follow-up (median 3.6 years after PET). No patients were lost to follow-up. The median time from HTx to PET was also long at 10 (6.7-16) years.

Limitations of the study include relatively small study population compared to prior published studies of the prognostic value of PET MBFR in HTx patients and the selected patient population studied (mainly comprised of patients with renal failure or other contraindications for standard surveillance with ICA).

The main findings were that survival probability was significantly higher in the high MBFR group compared to the low MBFR group, and that MBFR remained predictive of survival in the subgroup without previously documented CAV (but not in those with previously documented CAV).

Questions remaining to be answered include

(1) Can PET with MBFR serve as a safe and accurate alternative to ICA as the test of choice for routine serial surveillance for CAV after HTx?

(2) Can ICA be safely deferred if MBFR is normal?

(3) What is the optimal threshold for normal vs abnormal MBFR for assessment of CAV?
(4) Is a slight reduction in MBFR on serial surveillance testing meaningful? What percentage reduction in MBFR on serial testing should be considered predictive of the development of clinically relevant CAV?

Despite these remaining questions, it appears clear that cardiac PET with assessment of MBFR has emerged as a powerful tool for noninvasive serial surveillance of heart transplant recipients. Cardiac PET with assessment of MBFR is also widely recognized as a powerful tool for assessment of coronary microvascular disease. Given the accumulating clinical data and the outstanding safety and clinical feasibility, it seems to be only a matter of time before PET with assessment of MBFR is also used to guide patient management in hypertrophic cardiomyopathy, cardiac amyloidosis, aortic stenosis, and other clinical scenarios yet to be identified.

\section{Disclosures}

Dennis A. Calnon declares no financial conflicts of interest.

\section{References}

1. Bateman TM, Heller GV, Beanlands R, et al. Practical guide for interpreting and reporting cardiac PET measurements of myocardial blood flow: An Information Statement from the American Society of Nuclear Cardiology, and the Society of Nuclear Medicine and Molecular Imaging. J Nucl Cardiol 2021. https://doi.org/ 10.1007/s12350-021-02552-7.

2. Pelletier-Galarneau M, Dilsizian V. Microvascular angina diagnosed by absolute PET myocardial blood flow quantification. Curr Cardiol Rep 2020;22:9.

3. Sciagra R. Positron-emission tomography myocardial blood flow quantification in hypertrophic cardiomyopathy. J Nucl Med Mol Imaging 2016;60:345-61.

4. Bravo PE, Di Carli MF, Dorbala S. Role of PET to evaluate coronary microvascular dysfunction in non-ischemic cardiomyopathies. Heart Fail Rev 2017;22:455-64.

5. Wan N, Travin MI. Cardiac PET assessment of myocardial microvascular flow may help identify subclinical left ventricular dysfunction and increased risk from aortic stenosis. J Nucl Cardiol 2019;28:589-93.

6. Dorbala S, Vangala D, Falk R. Coronary microvascular dysfunction is related to abnormalities in myocardial structure and function in cardiac amyloidosis. JACC Heart Fail 2014;2:358-67.

7. McArdle BA, Davies RA, Chen L, et al. Prognostic value of rubidium-82 positron emission tomography in patients after heart transplant. Circ Cardiovasc Imaging 2014;7:930-7.

8. Bravo PE, Bergmark BA, Vita T, et al. Diagnostic and prognostic value of myocardial blood flow quantification as non-invasive indicator of cardiac allograft vasculopathy. Eur Heart $\mathrm{J}$ 2018;39:316-23.

9. Konerman MC, Lazurus JJ, Weinberg RL, et al. Reduced myocardial flow reserve by positron emission tomography predicts 
cardiovascular events after cardiac transplantation. Circ Heart Fail 2018;11:e004473.

10. Chih S, Chong AY, Erthal F, et al. PET assessment of epicardial intimal disease and microvascular dysfunction in cardiac allograft vasculopathy. J Am Coll Cardiol 2018;71:1444-56.

11. Feher A, Srivastava A, Quail MA, et al. Serial assessment of coronary flow reserve by rubidium- 82 positron emission tomography predicts mortality in heart transplant recipients. JACC Cardiovasc Imaging 2020;13:109-20.

12. Feher A, Sinusas AJ. Evaluation of cardiac allograft vasculopathy by positron emission tomography. J Nucl Cardiol 2021. https://doi. org/10.1007/s12350-020-02438-0.
13. Nelson LM, Christensen TE, Rossing K, Hasbak P, Gustafsson F. Prognostic value of myocardial flow reserve obtained by 82-rubidium positron emission tomography in long-term follow-up after heart transplantation. J Nucl Cardiol 2021. https://doi.org/10.1007/ s12350-021-02742-3.

Publisher's Note Springer Nature remains neutral with regard to jurisdictional claims in published maps and institutional affiliations. 